\title{
Taxonomy for Evaluation of Distributed Control Strategies for Distributed Energy Resources
}

Han, Xue; Heussen, Kai; Gehrke, Oliver; Bindner, Henrik W.; Kroposki, Benjamin

Published in:

IEEE Transactions on Smart Grid

Link to article, DOI:

10.1109/TSG.2017.2682924

Publication date:

2017

Document Version

Peer reviewed version

Link back to DTU Orbit

Citation (APA):

Han, X., Heussen, K., Gehrke, O., Bindner, H. W., \& Kroposki, B. (2017). Taxonomy for Evaluation of Distributed Control Strategies for Distributed Energy Resources. IEEE Transactions on Smart Grid, 9(5), 5185 - 5195. https://doi.org/10.1109/TSG.2017.2682924

\section{General rights}

Copyright and moral rights for the publications made accessible in the public portal are retained by the authors and/or other copyright owners and it is a condition of accessing publications that users recognise and abide by the legal requirements associated with these rights.

- Users may download and print one copy of any publication from the public portal for the purpose of private study or research.

- You may not further distribute the material or use it for any profit-making activity or commercial gain

- You may freely distribute the URL identifying the publication in the public portal 


\title{
Taxonomy for Evaluation of Distributed Control Strategies for Distributed Energy Resources
}

\author{
Xue Han, Kai Heussen, Oliver Gehrke, Member, IEEE, Henrik W. Bindner, Member, IEEE, \\ and Benjamin Kroposki, Fellow, IEEE
}

\begin{abstract}
Distributed control strategies applied to power distribution control problems are meant to offer robust and scalable integration of distributed energy resources (DER). However, the term "distributed control" is often loosely applied to a variety of very different control strategies. In particular there is a lack of discrimination between aspects related to communication topology, physical distribution of components and associated control objectives. This has lead to a lack of objective criteria for performance comparison and general quality assessment of state of the art distributed control solutions. For such comparison, a classification is required that is consistent across the different aspects mentioned above. This paper develops systematic categories of control strategies that accounts for communication, control and physical distribution aspects of the problem, and provides a set of criteria that can be assessed for these categories. The proposed taxonomy is applied to the state of the art as part of a review of existing work on distributed control of DER. Finally, we demonstrate the applicability and usefulness of the proposed classification to researchers and system designers.
\end{abstract}

Index Terms-distributed energy resource, distributed control, classification, control architecture, smart grid.

\section{INTRODUCTION}

G LOBAL efforts to replace fossil fuels with renewable energy sources [1]-[3] are driving a transformation of electrical grids and their operation. One aspect of this transformation is the proliferation of Distributed Energy Resources (DER), which include controllable and variable distributed generation, storage and controllable loads. This development creates challenges to power system operation, including to system stability, congestion management, voltage regulation as well as protection [4]-[8]. As conventional power plants - the traditional providers of operational flexibility - are substituted by smaller generators, DER units are increasingly in focus as possible providers of flexibility in a more and more actively managed grid [9]-[13].

Considering the increasing number of energy resources in the power system that will need to be controlled and coordinated, the complexity of a centralized control solution based on this traditional control paradigm is significant [14], [15]. The coordination of a large number of heterogeneous,

The major part of the work is supported by Danish national project iPower a Strategic Platform for Innovation and Research within Intelligent Electricity (SPIR), in collaboration with NREL.

Xue Han, Oliver Gehrke, Kai Heussen and Henrik W. Bindner are with the Department of Electrical Engineering, Technical University of Denmark (DTU), Denmark, Roskilde, DK-4000 e-mail: xueh, olge, kh, hwbi @ elektro.dtu.dk.

Benjamin Kroposki is with Power System Engineering Center, National Renewable Energy Laboratory (NREL), Golden, CO, 80401, USA. geographically dispersed and relatively unreliable units with spread ownership calls for novel control concepts which are structurally similar to the nature of the problem, i.e. which are distributed in their own right. Distributed control of DER units is a very active research topic within the context of Smart Grids, and a diverse range of applications and approaches have been proposed in a growing body of academic literature. Several attempts at analyzing and categorizing such control strategies have been undertaken for specific system types or application areas, such as applications in microgrids [16], electric vehicle (EV) charging [17] or demand response (DR) [18], for agent based systems [15], or for transactive control applications [19]. These works provide domain-specific categorizations from the perspective of a particular application, and adopt existing categorizations from one particular field such as computer science, control engineering or distributed systems. In the multi-domain, system-of-systems environment of smart grids, this leads to confusion because different subsystems of a given systems may exhibit different degrees of distribution, and because distribution is described in different ways by the different domains involved. As the Smart Grid domain matures, this is becoming increasingly problematic because the objectiveness of performance criteria is reduced if they are applied to a wide range of dissimilar technical solutions.

In Section II, the background about distributed control and existing categorizations in surveys are introduced to set the stage. To facilitate a systematic characterization of control strategies, we will introduce the classification method and criteria suitable for discriminating distributed control algorithms applied in smart grids in Section III. Next, as a key contribution of this paper, we develop a taxonomy of control strategies based on the above definition of distributed control, and provide a state-of-the-art review of distributed control strategies using the taxonomy (Section IV). It is followed by a case study in Section VI and further discussion in Section VII. The paper is concluded in Section VIII.

\section{State of the ARt of Categorization}

To assess the performance of a control solution, the reference for comparison would be a benchmark control problem and a set of criteria. For example, the problem of coordinating DERs to contribute to voltage control on a distribution feeder can be addressed in multiple ways: Using a hierarchical control system which collects measurements centrally and distributes setpoints, creating consensus between DER units through peerto-peer communication, or by local autonomous control, such 
as by following a droop characteristic. Each of these solutions is "distributed", however, their performance characteristics with respect to reaction time (control domain), resilience to communication failure (ICT domain) and optimality (e.g. with respect to loss minimization, power domain) are very different.

In this section, we review the current practice of conceptualizing distributed control systems as viewed from different domains, and discussed in other review papers.

\section{A. Domain-specific views on distribution}

Distributed control is inherently a multi-domain problem, and different criteria of distribution are used in the domains of power engineering, software, and control. When considering a problem which can only be solved by integrating subsystems from all three domains into an overall system, system-level characteristics of distribution will appear where the individual domains meet, in addition to the domains' own distribution characteristics. Two main characteristics are: Firstly, at the boundary between power system and control/automation, the division of software and (control) functionality between physical locations defines the way in which decisions are made, and which information needs to be exchanged in order to achieve the desired result. This architectural aspect defines for example whether raw data from a multitude of sensors is sent to a single software process for centralized evaluation, or whether local decisions are being made based on local data. Secondly, at the boundary between control/automation and computer science, the spatial distribution of information and communication infrastructure, i.e. the placement of computing units and the communication links between these units define the performance limitations of the decision making process. As an example, the master process in a master-slave control architecture could be executing on a dedicated master computer, or master and slave functions could be dynamically assigned between several identical computers.

The use of the term distributed control in the domains of automation and control dates back to the 1970s [20], [21], where it has primarily been applied to hierarchical master-slave systems in industrial automation. More recently, distributed control has also been used to refer to distributed control algorithms such as [22] where a distributed algorithm is used to control a physical system [23].

In computer science, a distributed system is defined as a collection of independent processes which appears to its users as a single coherent system [24]. The classification as a distributed system does not imply the use of a particular architecture for the implementation of its functionality; distributed systems may be hierarchical or not, be highly heterogeneous or be composed of identical processes. In nonhierarchical distributed systems such as peer-to-peer (P2P) systems, distributed algorithms are often employed in order to achieve a shared goal based on local decisions by individual communicating processes [25].

Power systems do not have a significant engineering tradition related to distributed control; the dominant system architecture combines autonomously operating, simple systems at the local level (droop controllers, breaker interlocks etc.) with master-slave remote control from a central location. The demand for a higher degree of automation, caused by the increasing complexity of the power system, requires a more integrated collaboration between the power systems domain and those of computer science, automation and control engineering [26]. Consequently, a more coherent understanding of distributed control is required which applies to such integrated systems.

\section{B. Categorization of Distributed Control in Surveys}

The state of the art of distributed control in smart grids has been surveyed from different angles, such as that of a specific power system application, implementation technique, or a specific control strategy (e.g. transactive control). Power system application areas relevant to distributed control of DER include EV charging [17], demand response (DR) [18] or microgrids [16], [27]. Most of these surveys provide a comprehensive perspective on grouping control solutions by their control objectives, such as [16], [17], [27].

Common classification concepts relate to control objectives. DERs contribute to grid services (instantaneous power; balancing energy) by adjusting active or reactive power, including: Power flow control and loss minimization by solving an optimal power flow problem for losses, aimed at limiting reactive and active power flow; Voltage and reactive power regulation by regulating reactive power injections (and active power in low voltage (LV) networks), to keep voltage magnitudes in an allowed band; Maximizing the utility of the resource operation, e.g., to minimize the overall charging cost of an EV fleet; Energy services, such as balancing the energy production and consumption, smoothing fluctuations, and avoiding temporary grid overload.

Another common power system application oriented classification is applied in [16], where control problems are categorized into primary, secondary and tertiary control levels (combining time scale, corresponding control objectives, together with a control hierarchy). A similar approach is found in [27] with three control layers. Reference is made to "communication", which appears to be a central argument with regard to the categorization of distribution types; however, as this criterion is then mixed with the distinction by control levels, so that this criterion is lost to the applicationoriented control levels. The distinction of distributed control strategies also mixes criteria from different domains, such as criteria of the software platform (e.g. agent-based) [15], of the mathematical approach to control algorithms (e.g. consensus, optimization decomposition) [17], [19], and of the distribution of controllers [17]. The software platform, agent-based, is the basis for classification in [15]; however, this is done somewhat incoherently across power system, ICT, or control applications and projects in the power system domain. In [17], distinction criteria include the mathematical formulation of the control objective, communication duplex and controller distribution. The survey provides an evaluation of control performance, discussing individual solution features, but does not provide a coherent view across all categories. 


\section{ClassificAtion FOR BENCHMARKING OF Distributed Control StRATEgIES}

As has been motivated above, existing categorizations of distributed control either take a single domain-specific view to achieve a coherent classification, or sacrifice coherence by mixing criteria from different domains. In this section the proposed classification method and criteria are motivated and developed.

\section{A. Properties of a Desirable Classification}

It is now clear that benchmarking of distributed control systems requires a view on multiple domains at once (here: ICT, control, power system). Yet, the engineering process for distributed systems is cascaded from application-domain requirements (power systems) through enabling domains (control, ICT), and engineering choices and design criteria tend to be domain-specific. As distribution aspects (in terms of locality, problem coupling, concurrency, communication and physical interactions) affect each engineering field, the allocation of solution components and interactions (as expressed in processing requirements, interaction protocols, sequences, APIs, ...), should be considered jointly.

A desirable classification will therefore, firstly provide a coherent view of this distribution structure, secondly formulate the relevant interactions affected by this distribution, and further thirdly identify evaluation criteria affected by all three domains:

- physical distribution of control problem and resources,

- distribution of the control algorithm and its interactions,

- ICT platform and implied communication requirements.

\section{B. Terminology}

The following terms conceptualize the distribution structure of a control strategy.

Controlled Process: A continuously operating, (cyber-) physical process (here: DER and/or power system) whose state is monitored and influenced by a control system.

Control Objective: A metric establishing the desired behaviour of a controlled process, which is to be achieved by an associated control system.

Control Element: A physical, spatially located, computing unit which is part of the control system, may interface with the process, and can execute (part) of the control algorithm.

Control System: A collection of interacting control elements that resolves a control problem by interfacing with a controlled process directly or indirectly through sensors and actuation (instrumentation).

Control Architecture: The spatial distribution and function of the entities in a control system, including control elements and instrumentation, and the relations between those entities.

Control Layer: A functional level in a layered control architecture, often representing the control problem at a particular time scale or abstraction level of the physical system.

Control Strategy: A collection of design choices pertaining the combination of control objectives, control architecture and control algorithm.
With the given terminology it is now possible to formulate the distribution structure of a control strategy as illustrated in Figure 1.

\section{Evaluation criteria}

Criteria for the selection or assessment of a control strategy can now be formulated with respect to the distribution structure. With benchmarking in mind, strategies must be categorized and be ranked by performance. A first categorical selection can be based on feasibility: Is, in principle, a given control problem aspect addressable with a specific distribution structure and set of interactions? The criteria corresponding to these feasibility aspects are referred to as Design constraints. They identify qualitative properties that need to be met by a control problem before the application of a particular control algorithm can be considered. Four apparent design constraints are defined in Table I.

Secondly, criteria for ranking the performance are formulated: assuming that a control problem can be addressed, how well does a specific control strategy perform on this type of control problem. A selection of performance metrics, which provide a quantifiable view of application requirements are listed in Table II.

Further important requirement categories have been identified, such as the design constraints 'cyber-security compliance' and 'market and regulatory compatibility', or the performance metric 'autonomy' (as a degree of independence from human intervention). Whereas these criteria are also relevant for the eventual choice of a control strategy, their evaluation cannot be based on distribution categories directly; evaluation results would be too problem-specific or implementation-dependent, rendering a general mapping between architectural properties and such evaluation criteria infeasible.

\section{TAXONOMY OF DISTRIBUTED CONTROL ARCHITECTURES}

Based on the concept of a distribution structure, common control strategies can now be categorized. A classification of distributed control architecture has to start by relating distributed architectures to their centralized and decentralized counterparts. Compared to a fully decentralized architecture, a distributed architecture requires direct communication between control elements; compared to a centralized architecture, the autonomy of remote control elements is increased.

The first classification criterion is therefore based on the structure of control elements:

T1: Centralized. One central control element collecting information from remote sites and deciding set-points for remote actuation; examples are [31]-[37].

T2: (D)istributed. Multiple control elements organized in a common architecture jointly responsible for decomposing objectives and deciding actuation.

T3: (Dec)entralized. A central (common) control objective is decomposed and delegated to independent local control elements; the local control elements only use local measurements and actuators; examples are [38]-[40]. 


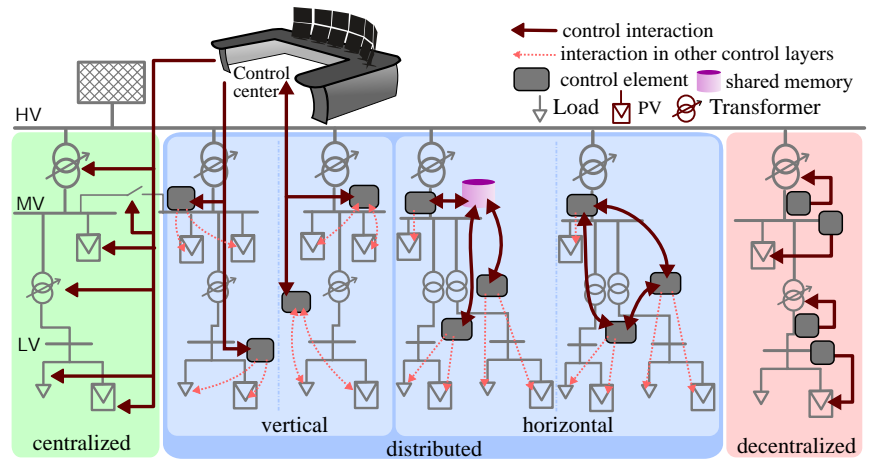

Fig. 1. Illustration of different interaction models (adapted from [4]). The figure excludes data acquisition subsystems. Large, solid arrows denote interactions within the control layer to be classified. Small arrows denote interactions in other control layers which are out of scope for the classification. The control center is an abstraction of the system operator (e.g. the SCADA/EMS/DMS functionality) and represents a central control element.

In the following, $T 2 D$ is classified further, based on the mutual roles of control elements in an architecture:

T2.1 D-(V)ertical. Decisions of one control element are imposed on other control element, eliminating at least one degree of freedom, so that a hierarchy among the control elements exists. Examples are [41]-[44].

T2.2 D-(H)orizontal. Different from T2.1 D-V the responsibilities of control elements in this category are symmetrical, and the functions being executed in the control elements are similar. Examples are [45]-[48].

The categories introduced so far are illustrated in Fig. 1 (centralized, distributed, and decentralized). For each of the distributed architecture types, two further subtypes are also illustrated in Fig. 1. The distinction criteria here are specific to each type:

For $T 2.1 D-V$ the control decision can be based on hierarchical information collection and central decision-making as it is common in conventional distributed process control, or based on an iterative negotiation process. The former case will be referred to as T2.1.A D-V-(D)eterminate; examples are [43], [44]. The latter will be referred to as T2.1.B D-V-(I)terative, as control decisions are made through iterative adjustments, and information is exchanged between higher and lower level control elements. This is indicated as bi-directional arrows in the figure; examples are [41], [42], [49].

While control responsibility is symmetric among T2.2 D$H$ architectures, an algorithm or coordination mechanism may or may not require a centralization of at least one aspect of information exchange. This centralization introduces a 'single point of failure' that would not exist in a fully $\mathrm{P} 2 \mathrm{P}$ architecture. In T2.2.A $D-H-(C)$ entralized shared memory, a single entity is available to relay information among control elements as an essential part of the control algorithm (e.g. [45], [46]). In contrast $T 2.2 . B D-H-(P) 2 P$ represents only fully distributed $\mathrm{P} 2 \mathrm{P}$ control architecture, as for example in [47], [48].

\section{ASSESSMENT OF DISTRIBUTED CONTROL ARCHITECTURES AND DER COORDINATION APPLICATIONS}

This section assesses each distributed control architecture by the alignment with the design criteria from Section III-C.

We first address the more common centralized and decentralized architectures, and then discuss the distributed control architecture variants in detail as the main focus of this paper. The results are summarized in Table III, which maps the properties of control architectures by category to the criteria discussed in Section III-C.

\section{A. Type 1: Centralized Architecture}

The structure of this type of control system is fairly simple, i.e., a single control element (control center) concentrates data collection, analysis and control of the entire controlled process. Significant computing and communication resources are required at the central location. This type of control algorithms is widely deployed in present power systems and typically referred to as Energy Management System (EMS) or Distribution Management System (DMS) as mature solutions. DERs directly react to control decisions derived from the EMS or DMS.

Information isolation cannot be realized because of the nature of data concentration, whereas this feature provides the control center the best overview of the controlled process. Therefore, it is possible to guarantee fairness, to obtain optimality, and to monitor the control actions from a single point in the system by using this architecture. Accordingly, a service that complies with a market with a centralized infrastructure can be formulated using this architecture, e.g., it is used in [33] to perform unit commitment in the existing energy market. Due to a simple structure, the control system is easy to maintain and its behavior is easy to interpret. As the intelligence is concentrated at a single point, the whole system cannot survive if the control center does not perform [13]. Similarly, the computation and communication capability at the control center limits the scale of such control system [15]. Responsiveness of this type of control algorithms is highly depending on the computation and communication capability,

TABLE I

DESIGN CONSTRAINTS

\begin{tabular}{lcl}
\hline Constraint & ID & Description \\
\hline \hline Information isolation and privacy & C-PRI & $\begin{array}{l}\text { Specific requirements on the limits of disclosure of behavioural and operational data between parties [28]. } \\
\text { Access to infrastructure and contributions to infrastructure cost is fairly shared by (distributed to) all } \\
\text { participants according to a given set of rules [29]. }\end{array}$ \\
Transactive energy framework & C-TEF & $\begin{array}{l}\text { A coordination scheme with a public specification of bi-directional signals, which transparently encode } \\
\text { value in exchange for resources in a manner aligned with a given operational and economic context. [11] } \\
\text { A consistent view of all control actions can be obtained from a single observation point in real time [30]. } \\
\text { It is necessary for operation supervision and supervisory control. }\end{array}$ \\
\hline
\end{tabular}


TABLE II

PERFORMANCE METRICS

\begin{tabular}{|c|c|c|}
\hline Metric & ID & Description \\
\hline Responsiveness & M-RTS & he time between observation of a change and actuation in the process. [17] \\
\hline Optimality and Accuracy & M-OPT & ifference between theoretically achievable global optimal/ideal outcome and practically achieved outcome. [17] \\
\hline Resilience & M-RES & $\begin{array}{l}\text { A measure of the capability to recover to stable system operation or adjust to changes due to disruptions or } \\
\text { unexpected disturbances [13]. }\end{array}$ \\
\hline Scalab & -SCA & Ability to continue meeting requirements while accommodating an increasing number of control elements. [15] \\
\hline Avails & -AVA & The degree to which the control system is in a specified operable state. \\
\hline Maturity & M-MAT & $\begin{array}{l}\text { Stage of development of the technology, which can be interpreted in terms of standardization, market adoption or } \\
\text { available technical experience. }\end{array}$ \\
\hline Ease & EOI & The ability of integration into existing workflows, processes and \\
\hline Maintainability & M-MTY & $\begin{array}{l}\text { The ease with which maintenance of a functional unit can be performed, which relates to the accessibility to } \\
\text { control infrastructure, including direct and remote access. [18] }\end{array}$ \\
\hline Operational transparency & M-OTR & xtend to which a human operator can infer the rationale decisions, actions and behaviour of an automatic system. \\
\hline
\end{tabular}

TABLE III

THE MAPPING OF TAXONOMY CATEGORIES AGAINST DESIGN CRITERIA.

\begin{tabular}{c|cccc|ccccccccc}
\hline Category & \multicolumn{9}{|c|}{ Constraints } & \multicolumn{7}{c}{ Metrics } \\
& PRI & FAI & TEF & MCA & RTS & OPT & RES & SCA & AVA & MAT & EOI & MTY & OTR \\
\hline \hline T1 Centralized & $\times$ & $\sqrt{ }$ & $\times$ & $\sqrt{ }$ & 0 & + & - & - & 0 & + & + & + & + \\
T3 Dec & $\sqrt{ }$ & $\times$ & $\times$ & $\times$ & + & - & + & + & + & + & + & - & - \\
T2.1.A D-V-D & $\times / \sqrt{ }$ & $\times / \sqrt{ }$ & $\times / \sqrt{ }$ & $\sqrt{ }$ & 0 & 0 & - & + & 0 & + & + & 0 & 0 \\
T2.1.B D-V-I & $\times / \sqrt{ }$ & $\sqrt{ }$ & $\sqrt{ }$ & $\sqrt{ }$ & - & 0 & 0 & 0 & + & 0 & 0 & 0 & 0 \\
T2.2.A D-H-C & $\times / \sqrt{ }$ & $\sqrt{ }$ & $\sqrt{ }$ & $\sqrt{ }$ & - & - & $0 /-$ & - & 0 & 0 & 0 & 0 & 0 \\
T2.2.B D-H-P & $\sqrt{ }$ & $\times / \sqrt{ }$ & $\times / \sqrt{ }$ & $\times$ & - & - & + & - & + & - & - & 0 & - \\
\hline
\end{tabular}

and therefore is scored " 0 " in Table III. Centralized control was employed in the Cell Control Pilot project [50].

\section{B. Type 3: Decentralized}

Decentralized control algorithms for power system applications are very common, such as in power/frequency droop serving primary frequency control or for local voltage control, e.g. with a reactive power/voltage droop. MetaPV [51] is a European practice of such architectural design that focuses on developing and demonstrating advanced control capabilities of PV inverters in a decentralized manner. Their design exploits inherent physical properties of the system, such as frequency being a global variable which reflects the system power imbalance. For example, the algorithms in [39] provide power factor or reactive power set-points as a function of local voltage magnitude.

In decentralized control algorithms, the control loop is closed locally and no internal information needs to be shared; further, no dependency on communication offers a basic resilience. Emergency actions and large-scale deployment are easy to realize using this type of control algorithm. The dependence on design-time parameters and assumptions may even cause severe incidents such as system collapse described in [4]. Coordination in decentralized algorithms is possible as control elements influence each other via physical properties of the controlled process, but not through direct interactions. As decisions are based on only partial knowledge of the relevant system state, this leads to suboptimal results. Also in this setup, trade-offs can be made between optimality and responsiveness in the design of control algorithms, e.g. by applying local signal processing and improved process models [52], [53]. Fairness is difficult to achieve directly, as it requires some system-wide coordination; in [52] a parametrization of local controls with topological information of DER connection points enabled more balanced DER contributions. Both monitoring of control actions and remote maintenance cannot directly be achieved due to the lack of communication. As the system information is concentrated nowhere but distributed along with control elements, it requires some efforts to understand how and why the control system responds to disturbances in the controlled process and to maintain the control system as such.

\section{Type 2: Distributed Architecture}

T2.1.A D(istributed)-V(ertical)-D(eterminate): A lot of dispatch applications use a vertically distributed architecture and communicate determinate set-points between control elements in different levels (see Fig. 2). The bottom level of the control system gains more autonomy compared to the type Centralized. Their control architecture is usually aligned with the hierarchical physical distribution grid structure, which makes this type of control algorithm easy to integrate. The control objectives are assigned to different levels in the control hierarchy. System level coordination objectives are placed on a higher level (e.g. coordination between multiple voltage levels [43], [44], between the grid and DERs [54], [55], and among various kinds of DERs [56] and grid assets [57], [58]), so that the fairness can be taken care of. Regional and local problems are handled in lower level(s) of the hierarchy. Control elements in this type of control algorithms organize the information associated with their corresponding problems, and exchange only relevant information with other control levels if demanded. The exchanged information can include a signal representing economic value. PowerMatchingCity [59] demonstrated the idea of PowerMatcher [60] using a $D-V$ $D$ architecture for providing several energy and balancing 
services. Grid4EU [61] aims at demonstrating advanced smart grid solutions with wide replication and scalability potential for Europe, one of which is a hierarchical control (D-V-D) strategies for load break switches in the field.

Since the information forwarded by lower levels is simplified and abstracted, the decision made in the high level may not derive system-wide optimal solutions with limited knowledge. For example, the set-points in the higher level are treated as part of the objective function at lower levels in [43], which cannot always be reached. In such case, suboptimal condition may occur. Abstraction of information in this control architecture also leads to lack of transparency on operational actions compared with centralized control solutions. Such architecture reduces the stress of the higher level control elements, both on exchanging and processing the in/out information, and on computation, but in the meanwhile limit the amount of information collected from a single point. Therefore, C-MCA is scored lower than Centralized, while M-RES and M-SCA are scored higher.

T2.1.B D(istributed)-V(ertical)-I(terative): Unlike $D-V-D$, in $D-V-I$ control elements on different control levels negotiate the decisions as illustrated in Fig. 3. A two level hierarchy is built in [7]: DER controllers handle the local control problem of deciding DER setpoints. The grid controller then negotiates with DER controllers to achieve a system wide solution. This allows lower level control elements higher level of freedom to decide on their contribution to the system; however, this approach sacrifices computational efficiency and system-wide optimality.

Game theory (e.g., [62], [63]) and optimization decomposition methods (e.g., [7], [41], [42], [49]) are two common mathematical methods to decompose such control problems. The values of auxiliary variables are iterated among sub-problems until convergence. The required information exchange is lim-

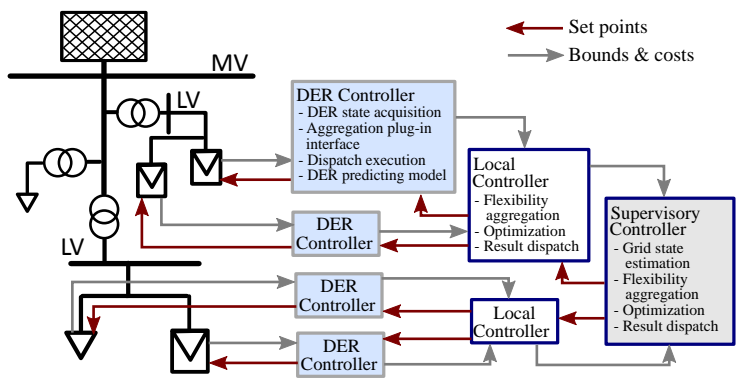

Fig. 2. An example using $D-V-D$ control architecture (adapted from [54]). The supervisory controller coordinates the whole network. The local controllers solve the sub-problems belonging to their regions. The DER controller focus on the operation of a single DER.

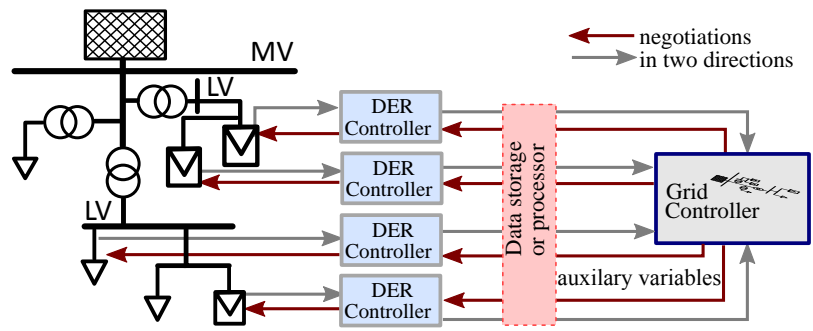

Fig. 3. An example using $D-V-I$ control architecture (adapted from [7]). Data repository or processor might exist in the architecture to process the data.

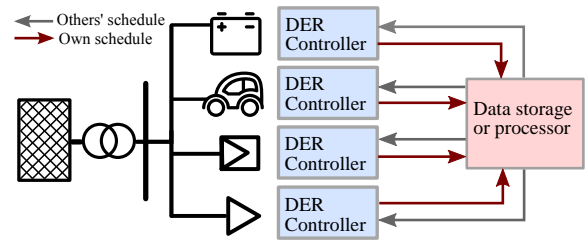

Fig. 4. An example using $D-H-C$ control architecture (adapted from [65]).

ited to algorithm-specific exchange variables, which avoids privacy concerns, but introduces difficulties on observing and understanding system behaviors. The applications applying this control architecture generally do not have quick response time, e.g., 1 hour is used as time interval in [7], [41], [42], [62], [63], because iterations of auxiliary variables take time to compute and to communicate. Given more freedom, the control elements at the lower level have more autonomy and take more responsibility than in the type $D-V-D$. Consequently, the control system is more resilient to partial failure, and is easier to maintain an operable state. However, none of the reviewed publications has performed any such analysis. DREAM project [64] proposed a control framework, in which distributed optimization $(D-V-I)$ is applied in a multi-agent environment to demonstrate its advance on information isolation to serve congestion management and voltage control.

T2.2.A D(istributed)-H(orizontal)-C(entralized shared memory): An optimization problem can also be decomposed to symmetrical sub-problems, i.e., equal responsibilities of control elements on decision making (see Fig. 4 and Fig. 5). For example, in [65], a distributed model predictive control algorithm is proposed on solving congestion problem at the point of common coupling. Instead of a centralized dispatch element, multiple DER control elements are designed for scheduling the consumption for individuals and sharing the efforts on not over-passing the capacity limit. Most applications in this category are motivated by a system-wide problem which requires a response from all connected units within a section of grid, such as in the case of power flow limitation [46], [65]-[67]. The behavior of each unit is impacted by all other ones.

A shared data repository collects (and processes) the data without manipulation with its own objective. In some cases (e.g., [65], [67]), information exchanges between all available control elements are avoided, so that the communication effort scales linearly with the number of control elements. However, communication with the central entity which may introduce bottlenecks and may limit the scalability. Since the data is collected at the shared memory, a single point access to all exchanged information is provided in this type of control algorithm, which makes it easy to monitor the control actions and to understand how a system behaves. All the control elements have symmetrical functionality, which makes every sub-problem more independent ones than in D-V-I. However, it may take longer time until all control elements agree on a set of solutions. Therefore, the responsiveness, optimality, and availability of such control algorithms are scored less than $D$ $V$-I. Privacy is algorithm and implementation dependent, as the shared memory includes all coordination-relevant data and presents a single point of attack. 
2.2.B D(istributed)-H(orizontal)-P(eer-to-peer): The control strategies of this category are fully distributed, such that all information exchanges between control elements are through P2P communication (see Fig. 5). The number of communication links increases with the number of control elements, which limits scalability. However, graph theory can be applied to reduce the number of communication links among control elements without losing much coordinating effectiveness. For example, a distributed optimal power flow algorithm was proposed in [68]: The distribution grid is divided into several regions and power flows are calculated locally for each region. Only control elements associated with neighboring regions communicate with each other by exchanging voltage information of interconnection buses between regions. The communication topology in [69] is designed according to a $\mathrm{N}-1$ criterion with redundant communication, such that an optimal dispatch is still available if one control element or communication link fails. Control time scales range from a few minutes to one hour. Some algorithms (e.g. [70]) may not guarantee convergence under all circumstances.

In this control architecture, all control elements are able to operate autonomously and communicate with others to obtain sufficient knowledge to support their decision making. In the proposed algorithms such as [48], [68], [71], there is no single point in this architecture where an overview of control actions, the state of the control system, and the state of the physical grid can be observed.

\section{Application of Taxonomy and Case Study}

The taxonomy and systematic review of distributed power system control strategies can support the assessment and selection of appropriate control algorithms. This section aims to illustrate how the taxonomy can be applied practice.

\section{A. Analysis of Hybrid Architectures}

In real-world solutions, a control problem can often be reformulated into several vertically layered control problems. The layer separation can employ time scale separation or other concerns. Each layer then can employ a different one of control patterns outlined above, forming a hybrid architecture.

The most common layering in power systems is the distinction of primary (I.), secondary (II.), and tertiary (III.) control layers. Here, the control problem is mathematically decomposed into a cascade, where each layer encapsulates certain disturbances while defining specific behaviour providing inputs to the next layer [72]. In conventional power system control, the layering is (I.-Dec., II.-Centralized, III.Centralized), but many alternative architectural realizations of this basic layering have been proposed [16]. In [73] a hybrid architecture mixes a Centralized with a Dec pattern for

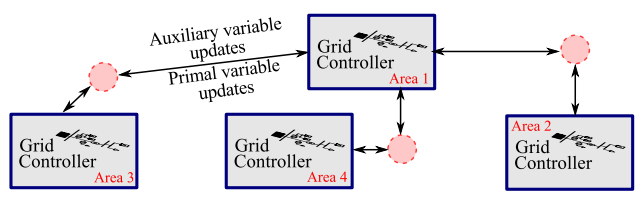

Fig. 5. An example using the $D-H-P$ control architecture.

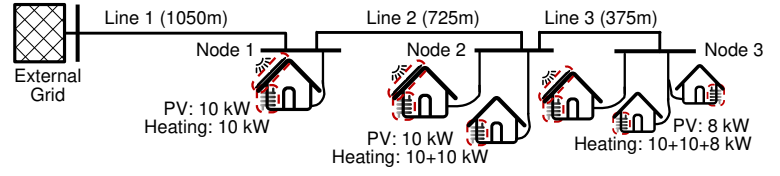

Fig. 6. LV distribution feeder used for the case study. Adapted from [54].

the coordination of photovoltaic (PV) inverters. Decentralized control elements are used for an inner control loop performing fast voltage support, while a centralized controller provides global coordination of the active power flow at the point of common coupling. A similar approach is proposed in [74]. [75] combines $D-V-D$ with a $D e c$ pattern in order to enable an efficient aggregation of flexibility while considering both global and local constraints.

The driver for layering different control patterns is typically the need to meet several design criteria that cannot be achieved in a desirable performance by a single integrated algorithm, or simply a separation of concerns to keep the overall architecture modular.

\section{B. Case Study}

We use a case study to illustrate a possible sequence of steps that can be used to formulate a control strategy. This case study is based on a control strategy proposed in [54], [76].

1) Identification of controlled process and flexibility: Controlled process: a residential LV distribution feeder with high penetration of PVs and electric space heating components in the laboratory as shown in Fig. 6. Flexibility constraints: PV active power production and curtailment is limited by solar irradiance; PV apparent power output is constrained by inverter capacity; Heating power consumption is constrained by indoor temperatures. Process constraints: Power flow constraints and power quality requirements.

2) Identification of control objective and available control elements: Control objective: The voltage magnitudes of all the nodes in the feeder should be within a band $\left[U_{\min }, U_{\max }\right]$, and the overall cost on activating flexibility from DERs should be minimized. This requires global snapshots of the system state space. Control elements: Computing resources of similar capacity and communication are available at all DER components.

3) Identify Criteria: As the background of the control strategy, the operating responsibility for the grid and of controlling DERs, each are assigned to Distribution System Operator (DSO) and aggregator, respectively (C-MCA). They are not supposed to disclose the grid operational information or DER status to each other. Therefore, the control strategy should be able to provide information isolation (C-PRI) between the units, aggregator and the DSO. Service delivery from aggregator to DSO should be verified by means of trusted grid measurements and the contribution of individual DERs are accounted for by the aggregator (M-OTR). To observe and enable trade-off across the primary purpose of DERs and to account for their contribution the aggregator requires C-TEF.

Voltage control requires immediate response to changes in voltage and power flows (M-RTS). Optimality is not critical, as 
temporary voltage rise/drop is acceptable within power quality limits (M-OPT). The objective quality contracted between the aggregator and the DSO should be met, and feasible setpoints should always be available for DERs (M-AVA). The control strategy should be able to adapt different kinds of events, such as addition of a new DER changes in DER flexibility due to subscription to a new service, communication link failure, etc (M-RES). Scalability is relevant for large-scale implementation (M-SCA). As part of the concern for largescale implementation, a generic flexibility interface should be ready for different types of DER technology, and different kinds of grid services (M-EOI, M-MTY).

4) Selection and evaluation of candidate architectures: Based on the identified requirements, Table III elucidates that Centralized and Dec cannot meet the full set of requirements. The distributed architectures (T2) D-V-D, D-V-I, or D-H-C may meet the design constraints, but appear to perform poorly on relevant metrics such as M-RTS or M-RES.

Decomposition of control problem into control layers: As $D-V-D$ satisfies most design requirements, except $M-R T S M$ $R E S$, and is the more mature of the candidates, it is selected. To meet responsiveness and resilience criteria, a three-layered approach is considered. The central (middle-layer), a $D$ - $V$ $D$ type control architecture is used to facilitate optimal and transactive coordination between DERs. At the bottom layer, decentralized droop controllers are used to let DERs track setpoints provided by the middle layer and respond to changes locally. To achieve resilience and architectural flexibility (MRES), a $D-H-P$ type control architecture is applied as toplayer, which renders the control hierarchy non-static as it can be renegotiated through an election process.

Control strategy: Layer 3: All DERs in the system are paired with identical control elements. The control elements can detect each others' presence (discovery) and interact to dynamically form a control hierarchy (election). Elections are triggered whenever the set of control elements changes. Layer 2 \& 1: Local controllers (LC), and a supervisory controller (SC) aggregate DER flexibility by means of a twolevel hierarchy, enabling the SC to coordinate the entire DER population and exchange dispatching information with the DSO.

Fig. 7 illustrates snapshots of control element activities during the election of a SC by LCs (left figure) and during control operation (right figure). Information flows from UCs via their corresponding LC to the SC which then submits flexibility (volume and cost) to the DSO. Flexibility requests by the DSO travel the other way.

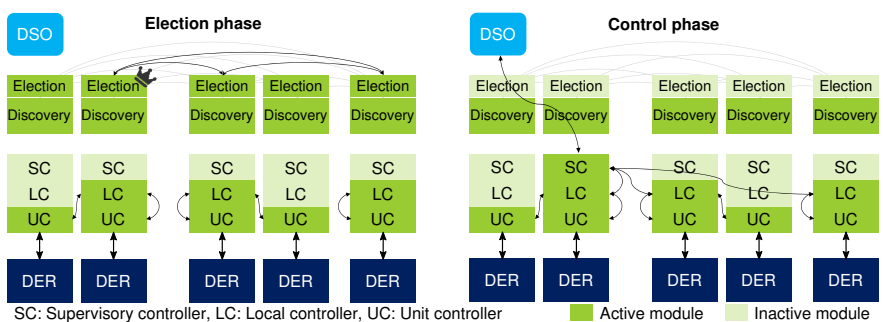

Fig. 7. Snapshots of control phases from the case study.

\section{DISCUSSION}

The analyses provided in this work lead to a few observations on the state of the art and expected future trends.

5) The present situation: Our qualitative meta-analysis in Section $\mathrm{V}$ enabled the evaluation of architectural approaches against the general criteria established in Section III-C, for which the results have been summarized in Table III. A reflection on the outcome provides a mixed picture: On first glance, traditional control architectures have clear and complementary properties; taken together, all of the considered constraints and performance metrics, except for C-TEF, can be met with either T1 Centralized or T3 Dec. On the other hand, the distributed architectures do not offer such clear-cut assessment. In summary, it appears natural that hybrid architectures combining Centralized and Dec systems are a common choice: the clear-cut separation establishes separated standards, design and engineering processes. For those requirements which are relevant for distributed energy resources, e.g. the need for scalable and resilient yet optimal resource allocation and control, these clear separations become a technical barrier for further development.

6) The benefits of distribution: The advantages of distributed architectures can then be summarized by two main points: a) the 'soft' advantage of architectural flexibility in meeting design objectives under consideration of other trade-offs such as privacy vs. fairness, or optimality vs. scalability; $b$ ) A Transactive Energy Framework (C-TEF) can only be realized using distributed architectures. The advantage of more suitable trade-offs across design criteria, however, implies a challenge to engineering processes which are more straightforward for traditional centralized/decentralized categories. It may be expected that the drive required to enable a transactive energy framework will facilitate the co-development of engineering processes and standards required to take advantage of the potential offered by distributed architectures.

7) Engineering practice: Another big challenge is the integration between distributed solutions and existing physical and information infrastructures. Related to the "Ease of integration" (EOI) metric in Table III, a simple insertion of distributed solutions into the existing power system infrastructure will not always yield an advantage because of the strong centralized tradition of the existing system on which the control solutions depend. A large effort is needed both on designing control strategies and on changing the mindset for system planning and operation.

8) The need for benchmarks: The last observation relates to an urgent need for benchmark models for smart grid solutions which could align the cross-domain knowledge and taxonomy in this field and measure the maturity of available approaches. In order to enable a fair comparison of control solutions, metrics from all involved domains as well as cross-domain metrics would have to be included. This would also help the development of engineering processes and standards. The categories and criteria provided in this work can offer a starting point for such an effort. 


\section{CONCLUSION}

The concept of distributed control has its roots in multiple engineering domains, all of which contribute to the increase in automation required for future electrical power grids. However, there is no general agreement on its definition in a power systems context. As a result, very dissimilar types of control strategies are labeled as "distributed", diluting the meaning of the term. This leads to difficulties when comparing control strategies. In this paper, a taxonomy and design criteria have been developed to assist in the design, selection, and evaluation of control strategies for DER applications with a focus on the control architecture. This contribution helps to establish a clear distinction between different approaches, which aids the design and evaluation of distributed control systems for specific applications.

Furthermore, this paper discussed the impact of the choice of control architecture on performance related to the design criteria. Based on the literature, strategies for the control of distributed resources were grouped by their categorization in the proposed taxonomy, and analyzed in terms of the proposed design criteria. The resulting architecture-based performance mapping can be employed in early steps of an engineering design process, and a case study illustrates how the method supports an informed choice of control architecture.

Future work will focus on two main aspects: Firstly, the qualitative analysis in this paper needs to be developed into a quantitative analysis of algorithm performance. However, this is a much larger undertaking which requires the development of benchmark models and scenarios including reference configurations for electrical network and control support infrastructure, as well as a specification of control problems broad enough to allow the comparison of algorithms. Different algorithms would then have to be implemented and executed in this reference environment, either as a (co-)simulation or in the laboratory. Secondly, this paper has focused strictly on the distributed nature of the control algorithm, ignoring distributed aspects of the ICT infrastructure required to execute the algorithm. Future work will need to illuminate this aspect as well. Finally, we provided a step toward systematic engineering processes required to facilitate the design and development of mature distributed control systems for power system applications.

\section{REFERENCES}

[1] European Commission. The 2020 climate and energy package. Last retrieved 20.05.2015. [Online]. Available: http://ec.europa.eu/clima/ policies/package/index_en.htm

[2] U.S. Federal Government. The American Recovery and Reinvestment Act of 2009. Last retrieved 20.05.2015. [Online]. Available: http: //energy.gov/oe/information-center/recovery-act

[3] International Energy Agency (IEA), "World energy outlook 2014," Tech. Rep., 2014. [Online]. Available: https://www.iea.org/publications/

[4] J. von Appen, M. Braun, T. Stetz, K. Diwold, and D. Geibel, "Time in the sun: the challenge of high PV penetration in the German electric grid," IEEE Power Energy Mag., vol. 11, no. 2, pp. 55-64, 2013.

[5] S. Achilles, S. Schramm, J. Bebic et al., "Transmission system performance analysis for high-penetration photovoltaics," National Renewable Energy Laboratory, Tech. Rep., 2008.

[6] Y. Liu, J. Bebic, B. Kroposki, J. De Bedout, and W. Ren, "Distribution system voltage performance analysis for high-penetration PV," in Proc. IEEE Energy 2030 Conf. Atlanta, GA, USA: IEEE, Aug. 2008.
[7] J. Hu, S. You, M. Lind, and J. Østergaard, "Coordinated charging of electric vehicles for congestion prevention in the distribution grid," IEEE Trans. Smart Grid, vol. 5, no. 2, pp. 703-711, 2014.

[8] L. F. Ochoa, C. J. Dent, and G. P. Harrison, "Distribution network capacity assessment: Variable DG and active networks," IEEE Trans. Power Syst., vol. 25, no. 1, pp. 87-95, 2010.

[9] "Buildings-to-Grid Technical Opportunities: Vision and Introduction," United States Department of Energy, Tech. Rep., Mar. 2014. [Online]. Available: http://energy.gov/eere/buildings/

[10] D. S. Callaway and I. A. Hiskens, "Achieving controllability of electric loads," Proc. IEEE, vol. 99, no. 1, pp. 184-199, 2011.

[11] The GridWise Architecture Council, "GridWise Transactive Energy Framework Version 1.0," Tech. Rep., Jan. 2015. [Online]. Available: http://www.gridwiseac.org/pdfs/te_framework_report_pnnl-22946.pdf

[12] F. Rahimi and A. Ipakchi, "Demand response as a market resource under the smart grid paradigm," IEEE Trans. Smart Grid, vol. 1, no. 1, pp. $82-88,2010$.

[13] C.-C. Liu, "Distribution systems: Reliable but not resilient?" IEEE Power Energy Mag., vol. 13, no. 3, pp. 93-96, 2015.

[14] M. Chiang, S. H. Low, A. R. Calderbank, and J. C. Doyle, "Layering as optimization decomposition: A mathematical theory of network architectures," Proc. IEEE, vol. 95, no. 1, pp. 255-312, 2007.

[15] P. Vrba, V. Marik, P. Siano, P. Leitão, G. Zhabelova, V. Vyatkin, and T. Strasser, "A review of agent and service-oriented concepts applied to intelligent energy systems," IEEE Trans. Ind. Informat., vol. 10, no. 3, pp. 1890-1903, 2014.

[16] M. Yazdanian and A. Mehrizi-Sani, "Distributed control techniques in microgrids," IEEE Trans. Smart Grid, vol. 5, no. 6, pp. 2901-2909, 2014.

[17] J. Hu, H. Morais, T. Sousa, and M. Lind, "Electric vehicle fleet management in smart grids: A review of services, optimization and control aspects," Renewable and Sustainable Energy Reviews, vol. 56, pp. 1207-1226, 2016.

[18] T. Samad, E. Koch, and P. Stluka, "Automated demand response for smart buildings and microgrids: The state of the practice and research challenges," Proc. IEEE, vol. 104, no. 4, pp. 726-744, 2016.

[19] J. S. Vardakas, N. Zorba, and C. V. Verikoukis, "A survey on demand response programs in smart grids: pricing methods and optimization algorithms," IEEE Commun. Surveys Tuts., vol. 17, no. 1, pp. 152-178, 2015.

[20] D. Popovic and V. P. Bhatkar, Distributed Computer Control for Industrial Automation. New York and Basel: Marcel Dekker, Inc., 1990.

[21] T. Pauly, "Distributed control systems," Electronics and Power, vol. 33, no. 9, pp. 573-576, 1987.

[22] R. Scattolini, "Architectures for distributed and hierarchical model predictive control - a review," Journal of Process Control, vol. 19, no. 5, pp. 723-731, 2009.

[23] D. P. Bertsekas and J. N. Tsitsiklis, Parallel and distributed computation: numerical methods. Prentice-Hall, Inc., 1989.

[24] A. S. Tanenbaum and M. van Steen, Distributed Systems, Principles and Paradigms. Upper Saddle River, NJ, USA: Prentice Hall, 2001.

[25] N. A. Lynch, Distributed Algorithms. San Francisco, CA, USA: Morgan Kaufmann Publishers Inc., 1996.

[26] M. Faschang, S. Cejka, M. Stefan, A. Frischenschlager, A. Einfalt, K. Diwold, F. P. Andrén, T. Strasser, and F. Kupzog, "Provisioning, deployment, and operation of smart grid applications on substation level," Computer Science-Research and Development, pp. 1-14, 2016.

[27] B. M. Eid, N. A. Rahim, J. Selvaraj, and A. H. El Khateb, "Control methods and objectives for electronically coupled distributed energy resources in microgrids: A review," IEEE Syst. J., vol. 10, no. 2, pp. 446-458, 2016.

[28] E. McKenna, I. Richardson, and M. Thomson, "Smart meter data: Balancing consumer privacy concerns with legitimate applications," Energy Policy, vol. 41, pp. 807-814, 2012.

[29] S. K. Vuppala, K. Padmanabh, S. K. Bose, and S. Paul, "Incorporating fairness within demand response programs in smart grid," in Innovative Smart Grid Technologies (ISGT), 2011 IEEE PES, Jan 2011, pp. 1-9.

[30] H. Gharavi and R. Ghafurian, "Smart grid: The electric energy system of the future," Proc. IEEE, vol. 99, no. 6, pp. 917-921, 2011.

[31] M. Juelsgaard, P. Andersen, and R. Wisniewski, "Distribution loss reduction by household consumption coordination in smart grids," IEEE Trans. Smart Grid, vol. 5, no. 4, pp. 2133-2144, 2014.

[32] I.-K. Song, W.-W. Jung, J.-Y. Kim, S.-Y. Yun, J.-H. Choi, and S.-J. Ahn, "Operation schemes of smart distribution networks with distributed energy resources for loss reduction and service restoration," IEEE Trans. Smart Grid, vol. 4, no. 1, pp. 367-374, 2013. 
[33] C. Zhao, J. Wang, J.-P. Watson, and Y. Guan, "Multi-stage robust unit commitment considering wind and demand response uncertainties," IEEE Trans. Power Syst., vol. 28, no. 3, pp. 2708-2717, 2013.

[34] S. Han, S. Han, and K. Sezaki, "Development of an optimal vehicleto-grid aggregator for frequency regulation," IEEE Trans. Smart Grid, vol. 1 , no. 1 , pp. $65-72,2010$.

[35] A. Keane, L. F. Ochoa, E. Vittal, C. J. Dent, and G. P. Harrison, "Enhanced utilization of voltage control resources with distributed generation," IEEE Trans. Power Syst., vol. 26, no. 1, pp. 252-260, 2011.

[36] N. Rotering and M. Ilic, "Optimal charge control of plug-in hybrid electric vehicles in deregulated electricity markets," IEEE Trans. Power Syst., vol. 26, no. 3, pp. 1021-1029, 2011.

[37] L. H. Macedo, J. F. Franco, M. J. Rider, and R. Romero, "Optimal operation of distribution networks considering energy storage devices," IEEE Trans. Smart Grid, vol. 6, no. 6, pp. 2825-2836, 2015.

[38] S. Steffel and A. Dinkel, "Absorbing the rays: Advanced inverters help integrate PV into electric utility distribution systems," IEEE Power Energy Mag., vol. 11, no. 2, pp. 45-54, 2013

[39] T. Fawzy, D. Premm, B. Bletterie, and A. Goršek, "Active contribution of PV inverters to voltage control-from a smart grid vision to fullscale implementation," Elektrotechnik und Informationstechnik, vol. 128, no. 4, pp. 110-115, 2011

[40] F. A. Viawan, A. Sannino, and J. Daalder, "Voltage control with onload tap changers in medium voltage feeders in presence of distributed generation," Electric power systems research, vol. 77, no. 10, pp. 13141322, 2007.

[41] D. Papadaskalopoulos, D. Pudjianto, and G. Strbac, "Decentralized coordination of microgrids with flexible demand and energy storage," IEEE Trans. Sust. Energy, vol. 5, no. 4, pp. 1406-1414, 2014.

[42] E. Dallr'Anese, S. V. Dhople, B. B. Johnson, and G. B. Giannakis, "Decentralized optimal dispatch of photovoltaic inverters in residential distribution systems," IEEE Trans. Energy Convers., vol. 29, no. 4, pp. 957-967, 2014.

[43] F. Delfino, R. Minciardi, F. Pampararo, and M. Robba, "A multilevel approach for the optimal control of distributed energy resources and storage," IEEE Trans. Smart Grid, vol. 5, no. 4, pp. 2155-2162, 2014.

[44] A. A. Aquino-Lugo, R. Klump, and T. J. Overbye, "A control framework for the smart grid for voltage support using agent-based technologies," IEEE Trans. Smart Grid, vol. 2, no. 1, pp. 173-180, 2011.

[45] A. Barbato, A. Capone, L. Chen, F. Martignon, and S. Paris, "A distributed demand-side management framework for the smart grid," Computer Communications, vol. 57, pp. 13-24, 2014.

[46] N. Forouzandehmehr, M. Esmalifalak, H. Mohsenian-Rad, and Z. Han, "Autonomous demand response using stochastic differential games," IEEE Trans. Smart Grid, vol. 6, no. 1, pp. 291-300, 2015.

[47] L. Xiao and S. Boyd, "Optimal scaling of a gradient method for distributed resource allocation," Journal of optimization theory and applications, vol. 129, no. 3, pp. 469-488, 2006.

[48] B. Zhang, A. Y. Lam, A. D. Domínguez-García, and D. Tse, "An optimal and distributed method for voltage regulation in power distribution systems," IEEE Trans. Power Syst., vol. 30, no. 4, pp. 1714 - 1726, 2015.

[49] O. Ardakanian, S. Keshav, and C. Rosenberg, "Real-time distributed control for smart electric vehicle chargers: From a static to a dynamic study," IEEE Trans. Smart Grid, vol. 5, no. 5, pp. 2295-2305, 2014.

[50] N. Martensen, H. Kley, S. Cherian, O. Pacific, and P. Lund, "The Cell Controller Pilot Project: Testing a smart distribution grid in Denmark," Proceedings of Grid Interpretability, pp. 216-222, 2009.

[51] Metamorphosis of Power Distribution: System Services from Photovoltaics (MetaPV). [Online]. Available: http://metapv.eu/

[52] P. J. Douglass, R. Garcia-Valle, J. Ostergaard, and O. C. Tudora, "Voltage-sensitive load controllers for voltage regulation and increased load factor in distribution systems," IEEE Trans. Smart Grid, vol. 5, no. $5,2014$.

[53] B. B. Johnson, S. V. Dhople, A. O. Hamadeh, and P. T. Krein "Synchronization of parallel single-phase inverters with virtual oscillator control," IEEE Trans. Power Electron., vol. 29, no. 11, pp. 6124-6138, 2014.

[54] X. Han, A. M. Kosek, O. Gehrke, H. W. Bindner, and D. Kullmann, "Activate distributed energy resources' services: Hierarchical voltage controller as an application," in Proc. IEEE PES Transmission\&Ddistribution Conference and Exposition 2014. Chicago, IL, USA: IEEE, Apr. 2014

[55] O. Kilkki, A. Alahaivala, and I. Seilonen, "Optimized control of pricebased demand response with electric storage space heating," IEEE Trans. Ind. Informat., vol. 11, no. 1, pp. 281-288, 2015.
[56] M. Bayat, K. Sheshyekani, and A. Rezazadeh, "A unified framework for participation of responsive end-user devices in voltage and frequency control of the smart grid," IEEE Trans. Power Syst., vol. 30, no. 3, pp. $1369-1379,2015$.

[57] X. Liu, A. Aichhorn, L. Liu, and H. Li, "Coordinated control of distributed energy storage system with tap changer transformers for voltage rise mitigation under high photovoltaic penetration," IEEE Trans. Smart Grid, vol. 3, no. 2, pp. 897-906, 2012.

[58] Y. P. Agalgaonkar, B. C. Pal, and R. A. Jabr, "Distribution voltage control considering the impact of PV generation on tap changers and autonomous regulators," IEEE Trans. Power Syst., vol. 29, no. 1, pp. 182-192, 2014.

[59] PowerMatching City. [Online]. Available: http://www. powermatchingcity.nl/

[60] J. Kok, C. Warmer, and I. Kamphuis, "PowerMatcher: multiagent control in the electricity infrastructure," in Proc. 4th Int. Joint Conf. on Autonomous Agents and Multiagent Systems. ACM, 2005, pp. 75-82.

[61] Grid4EU: Innovation for Energy Networks. [Online]. Available: http://www.grid4eu.eu/

[62] W. Tushar, B. Chai, C. Yuen, D. B. Smith, K. L. Wood, Z. Yang, and H. V. Poor, "Three-party energy management with distributed energy resources in smart grid," IEEE Trans. Ind. Electron., vol. 62, no. 4, pp. 2487-2498, 2014

[63] A.-H. Mohsenian-Rad, V. W. Wong, J. Jatskevich, R. Schober, and A. Leon-Garcia, "Autonomous demand-side management based on game-theoretic energy consumption scheduling for the future smart grid," IEEE Trans. Smart Grid, vol. 1, no. 3, pp. 320-331, 2010.

[64] Distributed Renewable resoures exploitation in Electric grids through Advanced heterarchical Management. [Online]. Available: http://www. dream-smartgrid.eu/

[65] G. T. Costanzo, O. Gehrke, D. E. M. Bondy, F. Sossan, H. Bindner, J. Parvizi, and H. Madsen, "A coordination scheme for distributed model predictive control: Integration of flexible DERs," in Proc. 4th IEEE PES Innovative Smart Grid Technologies Europe (ISGT-Europe'13). Copenhagen, Denmark: IEEE, Oct. 2013.

[66] C. O. Adika and L. Wang, "Non-cooperative decentralized charging of homogeneous households' batteries in a smart grid," IEEE Trans. Smart Grid, vol. 5, no. 4, pp. 1855-1863, 2014.

[67] H. Zhao, Q. Wu, Q. Guo, H. Sun, and Y. Xue, "Distributed model predictive control of a wind farm for optimal active power control part i: Clustering-based wind turbine model linearization," IEEE Trans. Sustain. Energy, vol. 6, no. 3, pp. 831-839, 2015.

[68] E. Dall' Anese, H. Zhu, and G. B. Giannakis, "Distributed optimal power flow for smart microgrids," IEEE Trans. Smart Grid, vol. 4, no. 3, pp. 1464-1475, 2013.

[69] W. Zhang, W. Liu, X. Wang, L. Liu, and F. Ferrese, "Online optimal generation control based on constrained distributed gradient algorithm," IEEE Trans. Power Syst., vol. 30, no. 1, pp. 35-45, 2015.

[70] S. Boyd, N. Parikh, E. Chu, B. Peleato, and J. Eckstein, "Distributed optimization and statistical learning via the alternating direction method of multipliers," Foundations and Trends in Machine Learning, vol. 3, no. 1, pp. 1-122, 2011.

[71] W. Zhang, W. Liu, X. Wang, L. Liu, and F. Ferrese, "Distributed multiple agent system based online optimal reactive power control for smart grids," IEEE Trans. Smart Grid, vol. 5, no. 5, pp. 2421 - 2431, Sep. 2014.

[72] K. Heussen, A. Saleem, and M. Lind, "Control architecture of power systems: Modeling of purpose and function," in Proceedings of the IEEE PES General Meeting 2009, 2009.

[73] M. J. E. Alam, K. M. Muttaqi, and D. Sutanto, "A multi-mode control strategy for VAr support by solar PV inverters in distribution networks," IEEE Trans. Power Syst., vol. 30, no. 3, pp. 1316 - 1326, 2014.

[74] S. Weckx, C. Gonzalez, and J. Driesen, "Combined central and local active and reactive power control of PV inverters," IEEE Trans. Sustain. Energy, vol. 5, no. 3, pp. 776-784, 2014.

[75] X. Han, H. W. Bindner, J. Mehmedalic, and D. V. Tackie, "Hybrid control scheme for distributed energy resource management in a market context," in Proc. IEEE PES General Meeting Conference \& Exposition 2015. Denver, CO, USA: IEEE, Jul. 2015.

[76] A. M. Kosek, O. Gehrke, and D. Kullmann, "Fault tolerant aggregation for power system services," in Proc. IEEE International Workshop on Intelligent Energy Systems (IWIES'13). Vienna, Austria: IEEE, Nov. 2013. 


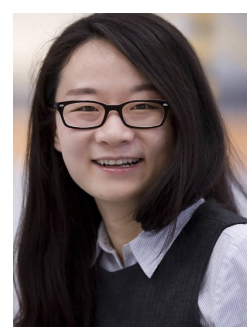

Xue Han is currently with the Center for Electric Power and Energy in the Technical University of Denmark as a postdoc researcher. She received the B.E. degree in electrical engineering and automation from Beijing Jiaotong University, China, in 2011, the M.Sc. degree from the Royal Institute of Technology, Sweden, in 2012, and the Ph.D. degree from the Technical University of Denmark, Denmark, in 2016. Her research interests cover the integration of renewable energy and management of distributed energy resources in smart grids.

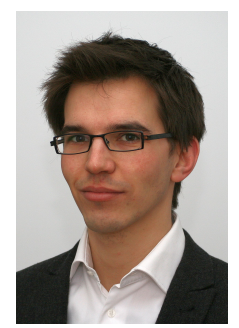

Kai Heussen obtained his Dipl.Ing. from the University of Stuttgart in 2007 and received his Ph.D. from the Technical University of Denmark (DTU) in 2011, where he is Assistant Professor at the Department of Electrical Engineering. His expertise is on demand side management, ancillary services design, automation, testing and verification in smart grids. His research interests include demand side management in distribution grids, control architectures for smart energy grids, and the design and development process of distributed control systems.

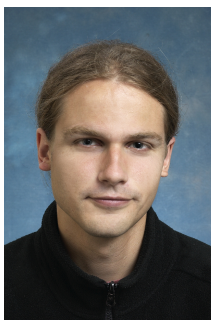

Oliver Gehrke received a Dipl.Ing. degree in Electrical Power Engineering from Darmstadt University of Technology in 2003. He started his career at the Wind Energy Department of RisøNational Laboratory, Denmark, working on the simulation of the control of hybrid renewable power systems and obtained a Ph.D. from the Technical University of Denmark in 2009. He is currently a Senior Researcher with the Technical University of Denmark. His main research interests are related to the embedded and distributed control of power systems with a high penetration of distributed energy resources, with an emphasis on experimental research.

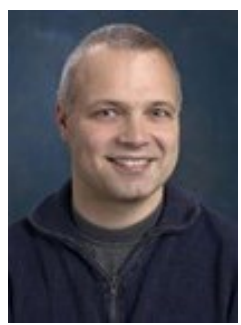

Henrik W. Bindner is a Senior Scientist and the head of Energy System Operation and Management research group in the Center for Electric Power and Energy (CEE) of Technical University of Denmark (DTU). He received the M.S. degree in electrical engineering from the Technical University of Denmark, Lyngby, Denmark, in 1988. Since 1990, he has been with the Risø National Laboratory for Sustainable Energy, Roskilde, Denmark. He has been focusing on developing technologies and control schemes enabling DER units to participate in the control of the power system. One of his main activities has been participation in establishing the experimental facility SYSLAB.

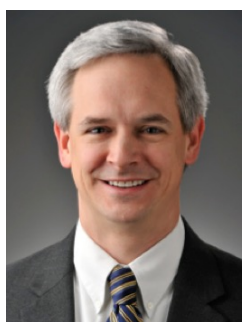

Benjamin Kroposki Dr. Benjamin Kroposki is the Director of the Power Systems Engineering Center at the National Renewable Energy Laboratory (NREL) where he leads NRELs strategic research in the design, planning and operations of electrical power systems. He received his BS and MS in Electrical Engineering from Virginia Tech and $\mathrm{PhD}$ from the Colorado School of Mines. His expertise is in the design, testing, and integration of renewable and distributed power systems and has more than 115 publications in these areas. As an IEEE Fellow, Dr. Kroposki was recognized for his leadership in renewable and distributed energy systems integration. He has served on a number of IEEE technical standards working groups and chaired IEEE 1547.4, the first international standard on microgrids. 